
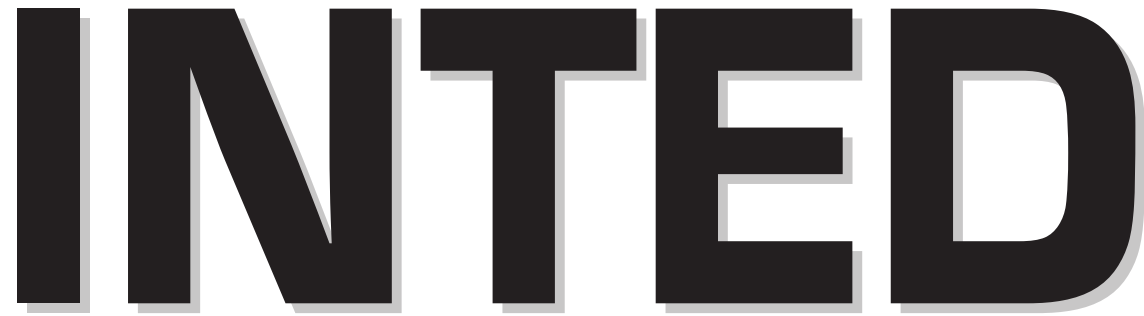

\section{7}

11th International

Technology, Education and

Development Conference

6-8 March, 2017

Valencia (Spain)

\section{CONFERENCE PROCEEDINGS}


Published by

IATED Academy

iated.org

INTED2017 Proceedings

11th International Technology, Education and Development Conference

March 6th-8th, 2017 - Valencia, Spain

\section{Edited by}

L. Gómez Chova, A. López Martínez, I. Candel Torres

IATED Academy

ISBN: 978-84-617-8491-2

ISSN: $2340-1079$

Depósito Legal: V-369-2017

Book cover designed by

J.L. Bernat

All rights reserved. Copyright (C) 2017, IATED

The papers published in these proceedings reflect the views only of the authors. The publisher cannot be held responsible for the validity or use of the information therein contained. 


\title{
TEACHING AND LEARNING ACTIVE PHYSICS WITHIN FRAMEWORK OF COMPETENCIES
}

\author{
E. Arribas ${ }^{1}$, S. Maffey ${ }^{2}$, R. Ramirez-Vazquez ${ }^{1}$, I. Escobar ${ }^{1}$, T. Franco ${ }^{2}$, S. \\ Vidales $^{3}$, C. Suarez ${ }^{3}$, J. Besanilla ${ }^{4}$, C. Garcia-Olguin ${ }^{4}$, A. Najera ${ }^{1}$, J. Gonzalez-

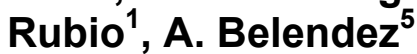 \\ ${ }^{1}$ University of Castilla-La Mancha, Albacete (SPAIN) \\ ${ }^{2}$ IPN, Ciudad de México (MEXICO) \\ ${ }^{3}$ UASLP, San Luis Potosí (MEXICO) \\ ${ }^{4}$ ENESMAPO, plantel $n^{\circ} 5$, Tamazunchale (MEXICO) \\ ${ }^{5}$ University of Alicante (SPAIN)
}

\begin{abstract}
The educational process, and as consequence, the student training in different disciplines is a topic of great interest for both national and international, such is the case in many of the science fields like Physics, reason why diverse institutions or organisms devote themselves to the task of studying the application of different implemented educational models. For example, the current educational model based on the approach for competences that appeared in the '70s, we will board this educational process from a completely theoretical point of view, trying to adjust it to Physics.
\end{abstract}

This educational model has a systemic approach that when applied to the teaching- learning process of a natural, factual, experimental and exact science like Physics, it implies that the knowledge to be considered should be relative to natural phenomena, demonstrable in an experimental form and that could be studied and modelled by means of mathematics. For such characteristics, the knowledge that constitutes it has been observed, studied and explained along the history, determining its conceptualization based on the knowledge that permit to adapt, to correct and/or clarify the vision and the knowledge in the different educational models.

The above mentioned model is based on the bonding that should exist between the formation and preparation, as well as the application of the knowledge, competences and skills in the student's job field, demonstrating with it the progress and successful learnings; obtaining with it the decision making. Nevertheless, it is important to consider that the results depend on several elements that are involved in the process, such as the teacher and the pupils; as well as, the different methods, tools, technologies and strategies that are implemented.

In a school environment, particularly, inside the educational model based on the approach for competences, we have focus on the task of studying and designing a methodological proposal for teaching and learning Physics within a framework of competences. This methodology can be explored and applied to different knowledge, educational levels, institutions, and why not, even after the combination of other methods and teaching techniques.

Keywords: Educational Model, Approach for Competences, Teaching-Learning Physics, Active Learning Physics.

\section{INTRODUCTION}

Education by competences is the educational model promoted at the dawn of the twenty-first century by UNESCO, considering it the methodological option that responds to the needs of the moment and in general terms, spanish and mexican educational policy adheres to it.

Argudin [1] affirms that educational competences are based on both the economy and the administration and try to approximate education to these disciplines, in an attempt to create better skills for individuals to participate in productive activity.

For the construction of skills, it is essential to develop skills, which involves locating knowledge, recovering it, transforming it and relating it to the knowledge already possessed to create or perform something effectively and efficiently. 
UNESCO's educational proposal for the present century is based on four principles, also called the "four pillars of education": learning to know, learning to do, learning to live together and learning to be [2], which are also stated as: knowing, knowing how to do, knowing how to live together and knowing how to be, so they are also known as the "four knowledges". In this paper the term "know" has a different connotation, taken from the didactic system proposed by Chevallard [3], in which a knowledge comes to be the knowledge to teach, to learn within a didactic system, as it was raised before.

\section{COMPETENCY EDUCATION}

To talk about education by competences we must start from the concept of competence, namely:

- UNESCO in 1999 defines competence as: The set of socio-affective behaviors and cognitive, psychological, sensory and motor skills that enable a performance, function, activity or task to be carried out properly.

- On the other hand, the National Association of Universities and Institutions of Higher Education (ANUIES) defines competency-based education as something that: It is based on a curriculum based on competencies in a comprehensive way and in solving problems. It uses resources that simulate the real life: analysis and resolution of problems, that deals in an integral way; Cooperative or team work, favored by tutorials.

- Chomsky [1] from the theories of language, establishes the concept and defines competence as the capacity and disposition for performance and for interpretation.

- Richard Boyatzis [1] states that a competence is the ability to demonstrate the sequence of a behavioral system that is functionally related to performance or to the result proposed to achieve a goal, and must be demonstrated in something observable.

- Frade [4] defines a competence as the specific capacity that has a subject to perform considering the demand that occurs in the environment and about which it has an intention.

- Argudín [1] concludes that a competence in education is a set of social, emotional and cognitive behavioral, psychological, sensory and motor skills that allow to properly carry out a role, a performance, an activity or a task.

Based on all of the above, this paper defines competence as:

A competence is the ability to perform specific tasks, which can be the same in everyday life, in the workplace or in the next educational phase, through a set of knowledge, skills, attitudes and values, acquired through a Formal educational process.

Nowadays it is necessary to abound in the education by competences or, in the generation of competences in the educative scope.

Education is a process that can be visualized in several ways:

- It is training.

- It is formation.

- It is teaching.

- It is learning.

- It is transformation.

The way in which the above five points are related is synthesized in the scheme of Figure 1. 


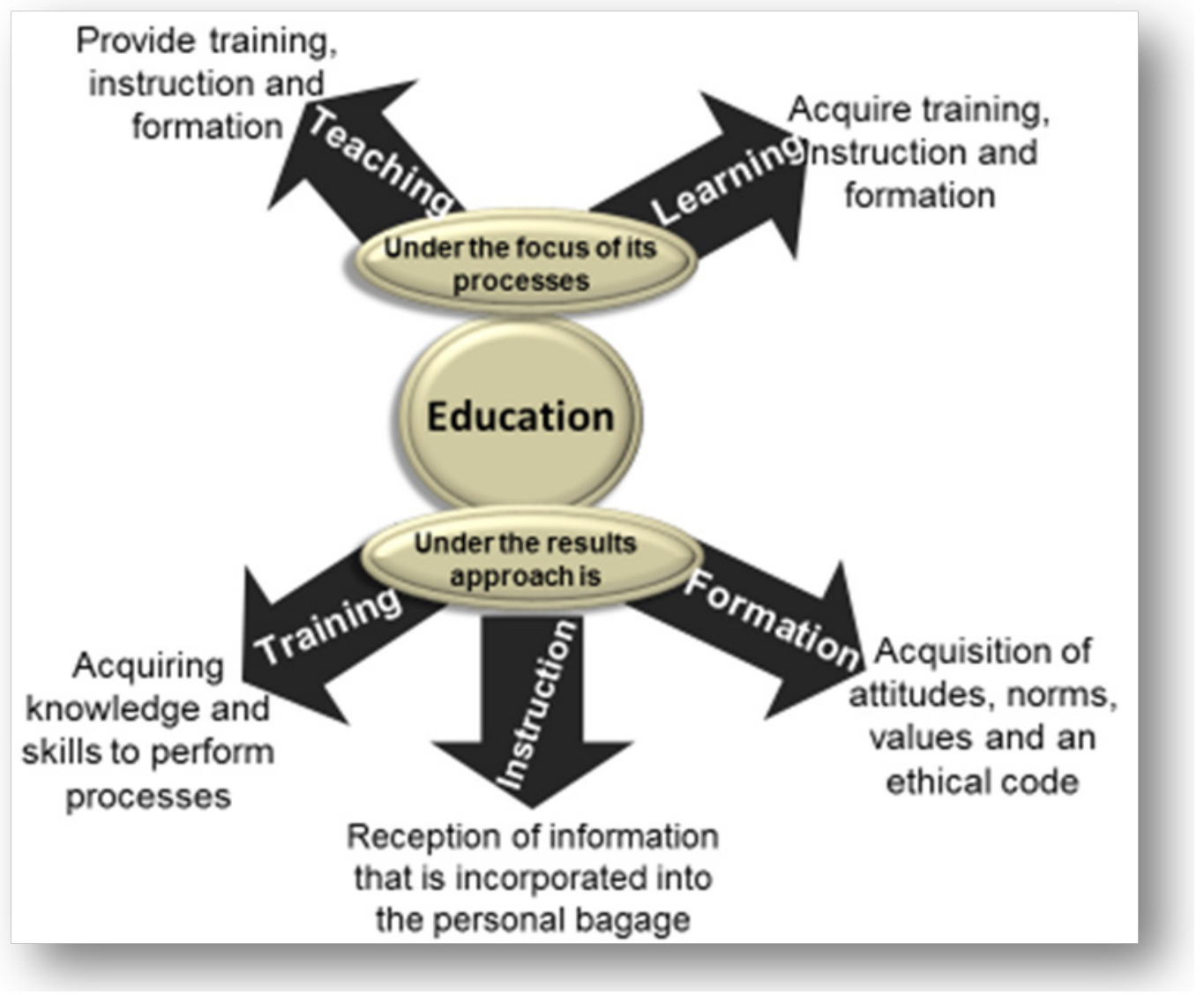

Figure 1. Education.

The main effect of any educational practice is that the student is educated, transformed, integrated into the social structure and the focus of the competences is to occupy a place in the economic production [1].

In competency based on education, the aim and the center of learning is the student and therefore it is necessary to reinforce the critical thinking of the student, so that the student has tools that allow him to discern, deliberate and choose freely [1].

In this educational modality, the work in the classroom is done through didactic sequences, which are designed with the aim of achieving the construction of a competition in the student. This requires a good theoretical basis, both in the teacher and the student, so that the latter, through the realization of the activities that are part of the didactic sequence, obtain an ideological structure that predisposes it to the growth and improvement of its performance [5].

The didactic sequence is designed according to the contents that lead to the construction of a certain competence, so it is used a relevant didactic situation in each case, which should be such as to motivate students to seek an explanation or solution, [6].

Theoretically, learning is achieved through a six-phase cognitive process, as presented by Frade [6], namely:

1 Sensation, which occurs when the five senses come into play.

2 Perception, which consists in making the sensation conscious.

3 Attention, it refers to that, of all the possible stimulus, is able to choose one, to concentrate on him.

4 Working memory, to remember what stimulus is, what it serves, what needs to be recovered from the information store itself to work with it. 
5 Conscious thinking to analyze, synthesize, make judgments, make decisions, create proposals, direct mental action and regulate behavior to elaborate and build what you want. This is the time when you really learn.

6 Performing a specific performance that takes a certain attitude, which is regulated by emotion.

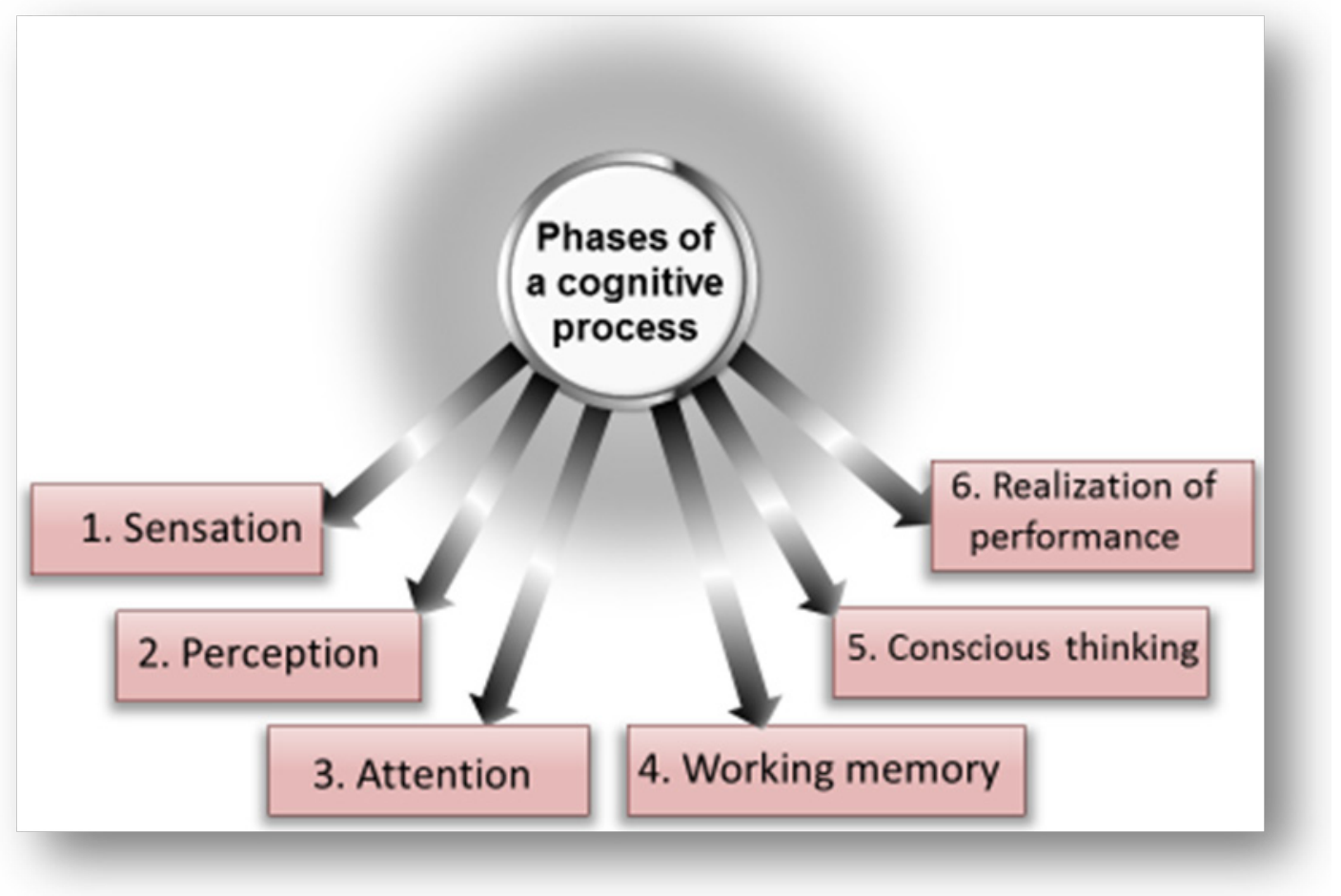

Figure 2. Cognitive process.

Finally, referring to the same author [6], it can be said that a competition has been built when the following points have been fulfilled:

- There is a constant interaction between people and the environment.

- In the process of learning successive actions are given, that is; antecedents and consequences are established, with which the subject develops the performance.

- Subjects in the process of learning, experience, recognize problems, look for causes and effects, in real life situations.

- Knowledge is used through multiple thinking skills, coming to analyze, synthesize, judge, make decisions and propose hypotheses.

- Applying the knowledge establishing priorities, interacting with the environment, and in this way is chosen.

Again appealing to Argudín [1], she states that in the 21st century, schools and organizations need to present a dual personality: learning the disciplines, developing disciplinary skills, generating an open learning environment with a curriculum flexible, with a certain stamp and offer the knowledge and skills that society requires.

Competences, like attitudes, are not potentialities that have to be developed because they are not given by inheritance or originate congenitally, but are part of the persistent construction of each person, his life project, what wants to carry out or build and the commitments that derive from the project that will perform.

From the curriculum, competency-based education focuses on:

- Knowledge.

- The abilities. 
- The attitudes inherent to a competition (ethical attitudes or behaviors that respond to discipline and values).

- The evaluation of the achievements by demonstrating the performance or the elaboration of a product.

Based on all of the above, the school curriculum must incorporate in its disciplinary contents: knowledge, skills, attitudes, values, strategies and methods, as well as evaluation mechanisms according to a performance philosophy. Each competition that is intended to build in an educator must be aimed at achieving something, to generate the capacity of performance of the latter in a context.

\section{PHYSICAL EDUCATION IN A COMPETENCY MODEL}

One of the difficulties inherent in the application of any pedagogical model is the transference of the underlying theory to the classroom, more specifically to the contents to work in the classroom. The case of physics is no exception.

The transit of traditional teaching models of physics, consisting of a more or less standard sequence: "theoretical explanation $\rightarrow$ mathematical modeling $\rightarrow$ problem solving $\rightarrow$ laboratory practice $\rightarrow$ practice report". New models that generate competences is not easy, even less when the teaching staff is made up of teachers with many years of work.

The change in traditional education in Physics towards a competency-based educational model must consider the application of new methodologies, as well as an in-depth analysis of the various aspects involved in the educational phenomenon.

Before speaking of competences, in the field of Educational Physics there was already an interest in finding methods, trends and strategies that would be able to efficiently learn the Physics, so the researchers, were given the task of reformulating the work of the teacher and the role of the student himself in the teaching-learning processes of this science, which led to the appearance of various proposals tending to overcome the results already mentioned.

To achieve this, different teaching-learning methods must be designed and implemented, some of which are shown in Figure 3.

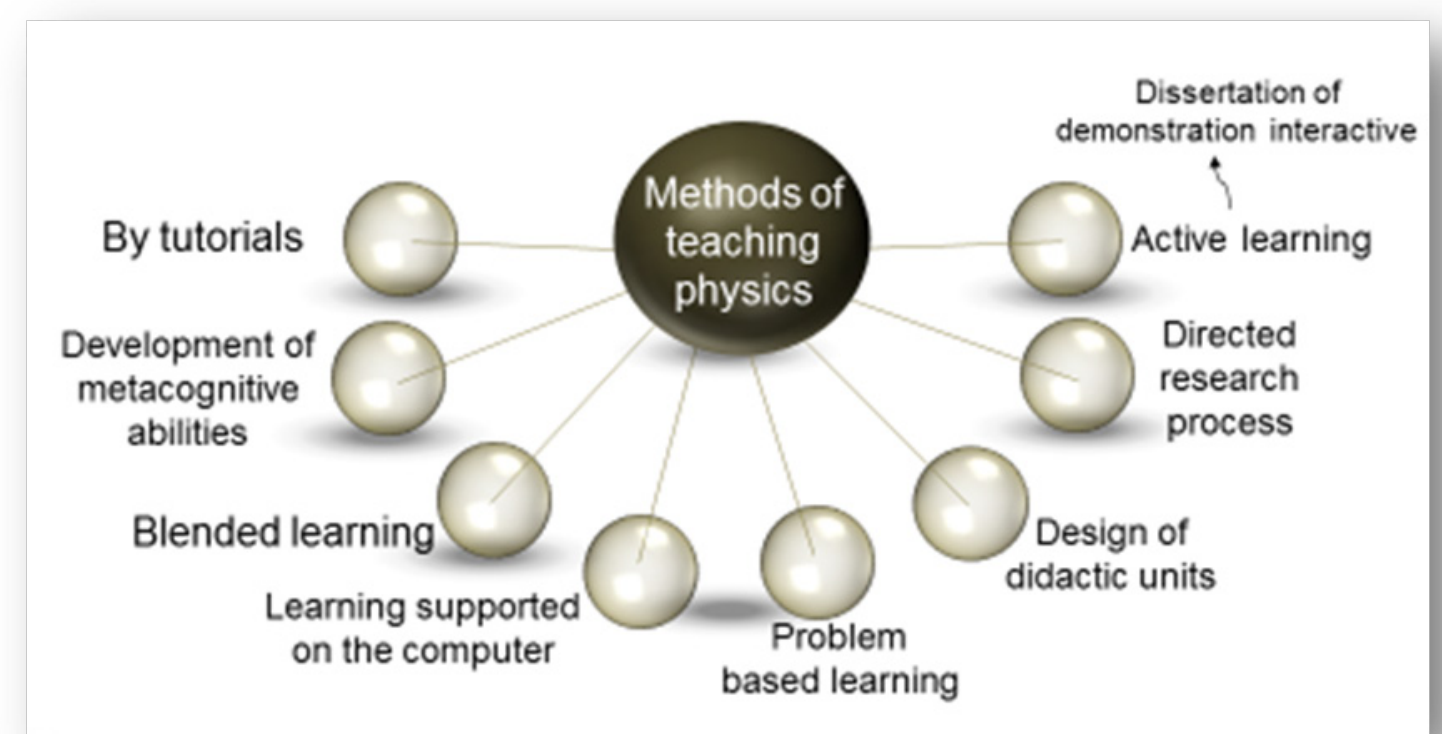

Figure 3. Methods of teaching Physics. 
However, after the methods have been designed and implemented, we proceed to the evaluation that allows to determine the competence of the student, so it is important to consider that when working under this approach in competencies, the evaluation is intended to measure performance, which involve conceptual, procedural and attitudinal contents. For this, Frade [4] mentions that it is necessary to design the evaluation as a process that is:

- Objective, without involving subjective criteria or personal judgments.

- Valid, that is, measure what you want to measure.

- Reliable, do not lend itself to different interpretations.

- Complete, considering all aspects or contents to be evaluated.

- Integral, including all areas involved in the proposed learning and all the knowledge, skills and attitudes that make it up.

- Significant, useful for the student, for the teacher and for the context.

- Leading to the decision making regarding the teaching - learning process that was carried out.

- Transparent, that is, containing auditable records.

Another aspect to consider during the process of global assessment of learning, is the multiple functions that are intended to achieve, such as:

- Determine the level of achievement of the students of the proposed learning.

- Feedback to students about their achievements and mistakes.

- Accreditation of students' performance, through the use of estimative scales.

- Diagnose the strengths and weaknesses of student learning and teacher education to reorient actions.

- Determine the relevance, timeliness, congruence and applicability of learning planning.

- Establish the efficiency and effectiveness of classroom work, teaching materials, resources and discourse, including the processes of metacognition, instruction and training.

- Plan the next course based on the experience gained.

- Provide the teacher with a clear basis for assigning grades or grades fair and representative of students' learning, achievement, and skill development.

With the competencies approach, the evaluation should be carried out in three stages, at the beginning of an educational process (initial or diagnostic evaluation), throughout the process (formative or procedural evaluation) and at the end of the process (summative evaluation). In the summative evaluation carried out at the end of the teaching-learning process, the reactants should evaluate both the student's competence in terms of specific knowledge, as well as the thinking skills and procedures required to answer questions or problems that arise.

Currently, a type of evaluation is proposed that includes a variety of evaluation techniques, understood as "any instrument, situation, resource or procedure that is used to obtain information on the progress of the process" [7].

Among the instruments of evaluation are two classes: observation techniques and performance evaluation techniques; The former serves as an auxiliary for the latter. The proposed instruments are shown in Figure 4. 


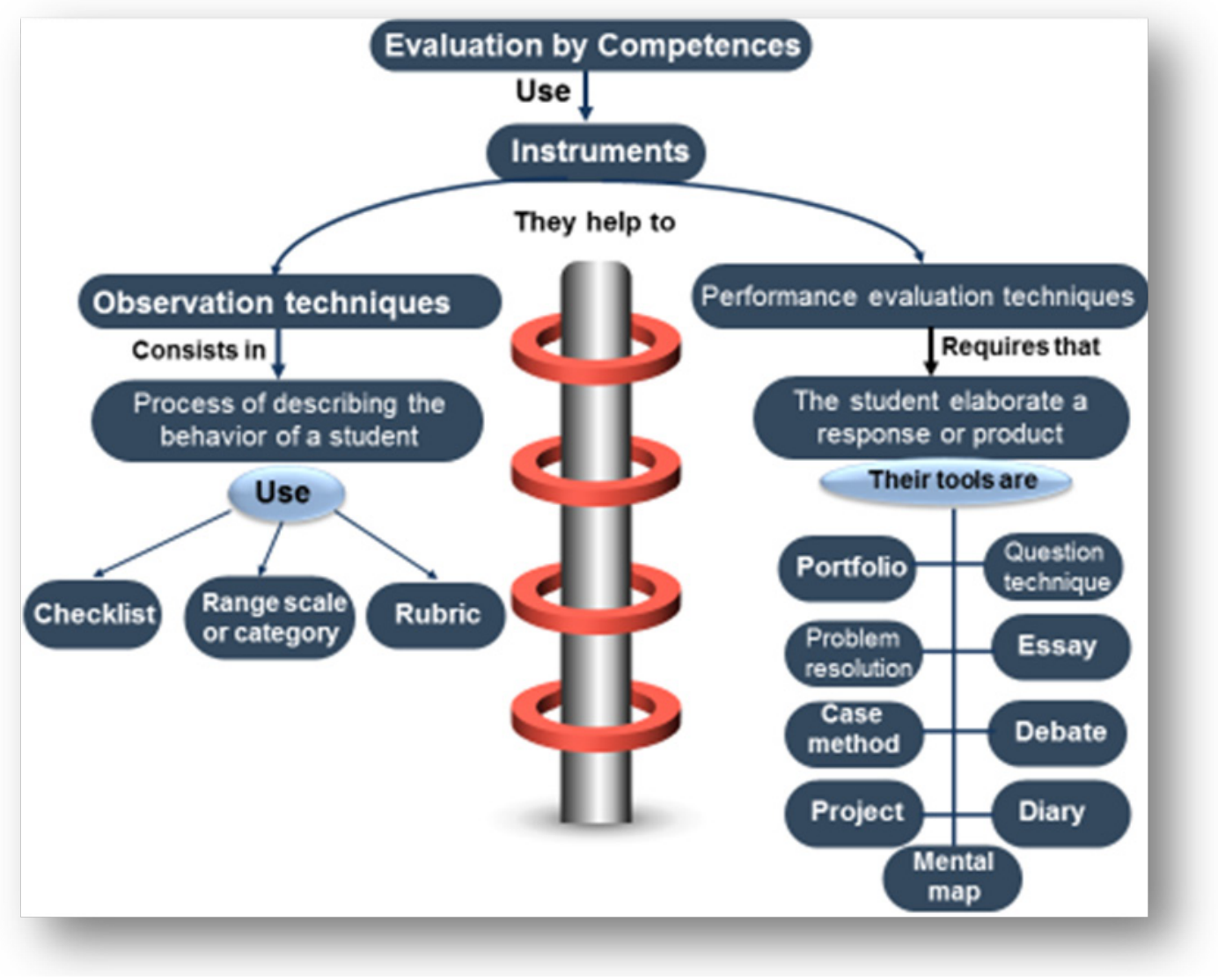

Figure 4. Instruments for the evaluation by competences of the Physics.

Observation as evaluation technique consists of a description process of behavior of a particular student, and the elements considered in that description are interpreted by the evaluator based on certain predetermined criteria.

Through observation, aspects and learning outcomes related to knowledge, skills, attitudes and values in different situations can be evaluated in an integral way, although it is used more frequently in the evaluation of contents of procedural and attitudinal type. For this purpose you can use:

- Checklist. It identifies behaviors and "must reflect the strengths and weaknesses of the students and promote a definition of goals" [7].

- Range scale or category. It consists of a group of characteristics that must be judged by a scale to determine the degree to which each is present.

- Heading. It is a table that has on the one hand the criteria that must be mastered and above are listed the ranges that will serve to evaluate the criterion domain. The numeric or verbal value assigned to each criterion must also appear.

On the other hand, the technique of performance evaluation is a method that requires the student to produce a response or a product that demonstrates their knowledge and skills. The techniques of execution are intended to evaluate what students can do, rather than what they know or feel. And thus, evaluate both the procedure employed and the resulting product, which can be compared with certain characteristics or with other products developed. Some of the tools considered in this area are:

- Portfolio. Its use allows the teacher and the student to monitor the evolution of the learning process and thus introduce changes during it, if it is necessary. It is a way of collecting information that demonstrates the skills and achievements of students.

- Problem solving. The problem solving process involves a set of skills that constitute that process and which is important to develop and evaluate in academic preparation. This technique revolves around a problem, which can be highly structured or unstructured. 
- Case Method. The evaluation with this method is done by reporting a situation that occurs or may occur in reality in a context similar to that in which students are or may be immersed and where they will make decisions. It allows to evaluate the probable form of performance of a student in a specific situation and at the same time, the way in which the participant puts into practice his ability to prepare written reports.

- Draft. A product is produced over a long period of time, with which, apart from demonstrating knowledge about specific issues, the ability to take responsibility, make decisions and satisfy individual interests can be evaluated. The main skill to be evaluated with this technique is to obtain information and organize it in a certain way so that it makes sense according to the objective set at the beginning of the project.

- Mental map. It is an image of the way in which the person gives meaning to a knowledge. It is a representation in diagram form that organizes a certain amount of information and with this one can evaluate the vision that the students have of the totality of a certain knowledge and of the relations between the information associated with that knowledge.

- Daily. It is an instrument that shapes the personal experience of each student during specific periods of time or activities. It is used mainly for self-assessment but can also be reviewed and considered by the teacher.

- Debate. It is a technique in which the teacher guides a discussion and observes the students' performance, noting the relevant aspects for the evaluation.

- Test. It is a written test of free response in which a student develops a subject freely expounding according to certain minimum criteria.

- Question technique. According to its design and the appropriate selection of questions to evaluate can reaffirm or develop schemes of thought in which the skills required for abstract reasoning are developed. The cognitive processes that are activated with the questions depend both on the content of the questions and on the context in which they are formulated.

After having talked about the educational model with a competency approach, as well as can be applied in the learning process of Physics, taking into account diverse teaching methods and tools, as well as the various evaluation instruments. It should be considered that the achievement of results will depend on the medium in which the system and the educational institution are applied, based on the governing documents, curriculum and curricula, methods of teaching physics already employed, teaching materials, teachers, students and how to evaluate it is used. Of all this will depend the efficiency, effectiveness and therefore the quality of the educational process.

\section{CONCLUSIONS}

The competency model currently implemented by educational institutions is playing a very important integrative role, and the positive or negative results depend on the entities involved and how they are applying the teaching-learning methods and the various evaluation tools, considering the usual methods and techniques and their understanding.

The implementation of this model has become a fundamental transformation axis for the teachinglearning process of the sciences, and in this case, for the teaching of physics. Since it is not a question of acquiring a new experimental culture, but rather of changing the experimental culture, constantly changing, adapting and improving the teaching-learning methods, tools and techniques used on a daily basis.

And as has already been reported, each of the elements involved in education, in this case in the teaching of physics or in any other discipline, play an important and determinant role in the results of educational action. Since an excellent method can be efficient with certain types of students suitable for a certain educational model and for others it may turn out to be all the same, it all depends on how and where it is being used.

\section{ACKNOWLEDGMENT}

Thanks to "Centro de Estudios Científicos y Tecnológicos No. 2 "Miguel Bernard" of the National Polytechnic Institute (Mexico), for allowing the application of the methodology proposed in this work. 


\section{REFERENCES}

[1] Y. Argudín, Competence-based education: notions and background. Mexico: Trillas, 2005.

[2] J. Delors, The education holds a treasure. México: Dower, 1997.

[3] Y. Chevallard, The didactic transposition. From wise to learned knowledge. Argentina: Aique, 1991.

[4] L. Frade, The Competence Assessment. Mexico: Quality Mediation, 2008.

[5] R. Tovar and G. Serna, 332 Strategies to Educate by Competencies. How to apply the skills in the classroom for high school. Mexico: Trillas, 2010.

[6] L. Frade, Competency planning. Mexico: Quality mediation, 2009.

[7] S. López and E. Hinojosa, Learning Assessment. Mexico: Trillas, 2002. 